\title{
The Sun as a Stellar Laboratory: Unsolved Problems
}

\author{
John C. Brown \\ Dept of Physics and Astronomy, University of Glasgow, \\ Glasgow G12 8QQ,Scotland, U.K.
}

\begin{abstract}
.
A brief overview is given of some of the current outstanding problems in solar physics with greatest emphasis on high energy phenomena in the atmosphere. The importance of plasma kinetic effects, as well as MHD, in understanding the complex finely structured and dynamic solar atmospheric plasma is stressed. Key results from the RHESSI Mission on energetic flare particle acceleration, propagation, and flare energy budgets are presented as are recent findings concerning the solar and stellar flare Neupert effect and the possible role of energetic particles in micro-events in the 'non-flaring' sun. Finally, evidence showing that magnetic fields are also important in hot star phenomena is mentioned.
\end{abstract}

\section{Introduction}

Having worked in both solar and stellar physics since around the time of the last IAU in Sydney, it was a pleasure and an honour to be asked to give a scene-setting talk at this Symposium. It comes at the end of a decade which has seen huge advances in both fields, especially in the areas addressed in this meeting. I have to confess at the outset that my stellar work is all on hot, not cool, stars but would caution strongly against the exclusive categorizations in which astronomers tend to indulge. Until recently it was widely believed that hot stars should not have magnetic fields but, as I will mention, recent data and theory show this to be quite untrue. So it is as well to keep our minds open to ideas from fields seemingly disjoint from our own. Magnetic fields appear to be ubiquitous in the universe and potentially omnipotent in their effects (magnetic fields being to astrophysics, Leon Mestel tells me, what Freud was to sex).

Recent instrumentation has taken stellar physics into the realms of precision probing ranging from astroseismology of interiors and rotation to plasma diagnostics of atmosphere and wind temperature, density and velocity fields, catching up on where solar physics was not long ago. Solar physics has meanwhile moved forward dramatically in this era of seismic mapping and neutrino probing of the interior, and of high resolution spectral imaging of the outer layers, the latter now spanning the electromagnetic spectrum up to gamma-ray lines. Probing of the interplay between the sun and the interplanetary medium has advanced via in situ probes, 3-D mapping (Ulysses), and observations of CME propagation. 
In 1991, Joan Schmeltz and I directed a NATO ASI on The Sun: a laboratory for astrophysics, many students from which were at Symposium 219 as today's leaders in solar physics. Twelve years on is a fitting time to be taking a look in that vein at progress and unsolved problems in studies of the sun and its relation to stars.

\section{Major Problems of Solar Physics}

It is possible here only to touch briefly on a few of the many problems still posed by the sun and to elaborate a little on a select few of special interest to me and with links to topics addressed in subsequent papers of these proceedings. In the broad brush view we can divide solar physics problems into those of the interior and of the exterior. There are of course crucial issues at the photospheric interface like variability of the radiative output, linking interior dynamo theory to the structure of sunspots and active regions, and the effect of active regions on the surface appearance of helioseismic waves.

As far as the interior is concerned there have been huge advances in the key problems of :-

- the neutrino flux paradox, essentially solved in terms of neutrino oscillations (e.g. Ahmad et al. 2002)

- the interior structure, including rotation and even its time variations, via helioseismology though the g-modes and hence the core structure remain elusive (e.g. Gough 2000)

- the dynamo (Weiss 2002; Weiss and Tobias 2002) in terms of convection and MHD theory (e.g. Thomas et al. 2002) and its relation to the complex surface magnetic structure (e.g. Scharmer et al. 2002)

As to the atmosphere and beyond, the following major problems are still unsolved despite much progress in many aspects of them :-

- the nature of chromospheric heating and the consequent evaporation, through radiative instability, of material into loops, the general corona, and the wind

- the nature of coronal heating, presumably via reconnection

- the extent to which the above involve localized transient events as opposed to quasi-steady processes

- the fundamental nature of the fast reconnection required in flares

- the processes of electron and ion acceleration

- the role of fast particles in flare energy transport

- the mechanisms involved in CMEs and their relation to flares

- space weather and sun-earth interactions 


\section{The Importance of Particle Kinetics in the Solar Atmospheric Plasma}

In contrast to the magnetosphere/space plasma community, solar physics has put by far the greatest emphasis on MHD. While this approximation may be fine for many gradual and large scale phenomena, its limitations need to be more fully appreciated when dealing with transient and small scale processes. The ever more ubiquitous occurrence of such processes revealed by successively better solar instruments, not only in flares but in the 'quiet' sun (moss, bright points, blinkers, micro-events etc) emphasizes the need for thinking beyond the pure MHD approach.

In the case of flares, the need for a inclusion of plasma kinetics is easily seen from simple theoretical considerations based on the most rudimentary observations. Release of the magnetic energy of a field $B$ into a plasma of density $n$ yields a mean particle energy (neglecting losses)

$$
\bar{E}=\frac{B^{2}}{8 \pi n} \approx 25 \mathrm{keV} \frac{B_{2}^{2}}{n_{10}}
$$

where $n_{10}=n / 10^{10} \mathrm{~cm}^{-3}$ etc. The collisional mean free path of even such mean particles is huge compared to the length scales required for current sheets and shocks to allow the fast reconnection in flares. Furthermore the total number of such particles involved in the volume needed to release total energy $\mathcal{E}$ is

$$
\mathcal{N}=\frac{\mathcal{E}}{\bar{E}}=2.5 \times 10^{38} \frac{\mathcal{E}_{31} n_{10}}{B_{2}^{2}}
$$

which is comparable to the number of $\geq 20 \mathrm{keV}$ electrons known to reach HXR footpoints in chromospheric flares. Thus essentially all of the electrons in the magnetic energy release volume have very long mean free paths and are seen directly to leave the reconnection region over tens of seconds at a rate fluctuating in time scales $\leq 1 \mathrm{sec}$. This situation is far removed from the MHD approximation which suggests that omission of kinetic effects in most, though not all, theoretical treatments of reconnection may be one factor in the difficulty of obtaining fast enough reconnection. In addition to their long mean free paths, the high beam currents carried by the fast particles means that they, and their neutralising plasma return currents, cannot be ignored in the global electrodynamics of reconnection (cf Litvinenko 2000; Petkaki and MacKinnon 1997).

\section{Recent Results on Flare Particles}

In view of the importance of plasma fast particle kinetics argued in Section 3, here I will highlight what I see as some of the most exciting new results obtained over the past eighteen months concerning them by the RHESSI mission (Lin et al. 2002), an overview of which is also in these proceedings (Lin 2003) together with a description of the instrument. 


\subsection{Hard X- and Gamma-ray Spectrometry}

The advent of Ge detectors has advanced spectrometry of high energy photons (energies $\epsilon \approx 1 \mathrm{keV}$ to $10 \mathrm{MeV}$ ) from scintillation counter resolutions $\epsilon / \Delta \epsilon$ of order 3 to the range $10-1000(\Delta \epsilon \approx 1 \mathrm{keV})$. This has enabled resolution of nuclear gamma-ray line profiles (Smith et al. 2003) and provided hard X-ray continuum data with a resolution sufficient to allow, for the first time, practical implementation of the spectral inversion approach. This was first advocated by Brown (1971) and developed into practical numerical schemes by Craig \& Brown (1986), Thompson et al. (1992), Johns \& Lin (1991), Piana (1994) and Piana, Brown \& Thompson (1995). The philosophy of this approach is to derive the HXR source mean electron flux spectrum $\bar{F}(E)$, discussed by Brown(1971), Johns \& Lin (1991) and Brown, Emslie \& Kontar (2003), from the HXR photon flux spectrum $I(\epsilon)$ without any parametric assumptions about the spectral form other than applying an a priori constraint of smoothness (bin-widths in the case of Johns \& Lin) to control the effects of data noise magnified by the inversion. First results from RHESSI data are to be found in, Conway et al. (2003), Massone et al. (2003) and Piana et al. (2003)the last also comparing results with those (Holman et al. 2003) of forward parametric fitting. The results show a number of hitherto unknown features, the significance of which is under investigation. They include cases (Kontar, personal communication) where the spectrum seems to be almost power-law at all observed energies with no exponential (isothermal) component, or power-law with exponential cutoff. In others $\bar{F}(E)$ can be interpreted purely thermally with a suitable emission measure distribution $D E M(T)$. (The idea that $\bar{F}(E)$ cannot be Maxwellian to very high $E$ because the collisional mean free path is too long does not necessarily hold since a distribution can be Maxwellianized by interaction with waves). The potentially most exciting feature found in $\vec{F}(E)$ is a pronounced dip around $50 \mathrm{keV}$ (Piana et al 2003; Johns \& Lin 2003). If real, this could have key implications for electron acceleration mechanisms but, at the time of writing, there is still a suspicion that the corresponding feature in the photon spectrum may be associated with detector pulse pile-up. However the pile-up would have to be of a magniture larger than expected even at the comparatively large flux levels of the event concerned. The disappointing pile-up interpretation gains some credibility since the feature is in an energy range where a pile-up effect is expected to be significant. On the other hand, it is, perversely, also near the energy where the effects of photospheric backscatter (Alexander \& Brown 2003) and of atmospheric ionisation structure (Kontar et al. 2003) are largest, which compounds the complexity of interpretation.

\subsection{Hard X-ray and Gamma-ray Spectral Imaging}

RHESSI spectral images of flares reveal structures considerably more complex than seen hitherto and with a diversity of morphologies. Since chromospheric HXR footpoints are markers of field lines from the coronal reconnection and acceleration site, these morphologies are important clues to the reconnecting field topology. However, in many cases the main HXR image features seem to bear out the canonical collisional thick target model (Brown 1971) predictions of two bright loop footpoints and faint upper loop emission, the brightness ratio $\gg 1$ depending on photon energy (and spectral index), as well as on coronal 
density, as expected (e.g. MacKinnon et al. 1986). Aschwanden, Brown \& Kontar (2002) found that the HXR footpoint centroid altitude $h$ decreases with increasing photon energy, as expected qualitatively and, using the theory of Brown, Kontar \& Aschwanden (2002), inferred the height distibution $n(h)$ of atmospheric density which would be required for these data to be consistent with the predictions of the collisional thick target model. They found the resulting $n(h)$ to be reasonable though considerably higher than $n(h)$ in spectroscopic models of flare atmospheres and closer to the values measured in spicules. In this interpretation the electron beam is assumed to be collision dominated and to have a power-law injection spectrum, and is used as a probe of $n(h)$. One could, however, alternatively assume $n(h)$ from other data to be correct and use the observed deviation from collisional thick target predictions of footpoint centroid heights as an indication of non-collisional energy losses (e.g. Brown \& MacKinnon 1985; Haydock et al. 2001; Emslie, Barrett \& Brown 2001).

Not all flares adhere to this simple characterization, however. Some nearand behind-the-limb events show substantial and long-lasting emission at high altitudes (Kane 2003) while Veronig \& Brown (2003) have discovered a seemingly new class of event in which most of the HXR emission is in the upper loop rather than the footpoints. This is because the upper loop in these is so dense (as measured by GOES SXR emission measure) that it constitutes a collisionally thick target for electrons up to around $60 \mathrm{keV}$ which thus never reach the loop footpoints. These occulted-event sources and coronal thick target loop sources both seem to be quite distinct from the relatively faint 'above-the-looptop' HXR sources found by Masuda (1994)

Fletcher \& Hudson (2002) have shown that the centroids of RHESSI HXR footpoints move with time, presumably reflecting topological changes in the field lines linking the reconnection site to the chromospheric footpoints. At any one time, therefore, the HXR sources do not occupy the entire ribbon area swept out during the flare as supposed in early work. The HXR resolution in this study is rather modest at around 8" but the centroids of the HXR footpoints seem to track the XUV footpoints seen by TRACE at a resolution of order 1". If the HXR sources were really as small as the TRACE ones then a thick target electron beam model could be untenable since the plasma return current drift speed would have to exceed the ion-sound speed and possibly even the electron thermal speed. This situation would be highly unstable to wave generation and dissipate the beam, unless large pitch angles reduced the beam current or the beam flowed in very hot narrow channels within the area. Based on the smallest HXR source areas observed by RHESSI, Veronig et al. (2003) found that the situation is very close to the electron thermal speed limit, but not over it, again depending on the value of spectral cut off $E_{c}$. Consequently, return current instability may, as suggested by Brown \& Melrose (1977) be acting to limit the accelerated electron flux.

As regards ions, the first solar imaging spectroscopy at gamma-ray line energies by RHESSI (Hurford et al. 2003) has yielded the profound result that the source of the $2.2 \mathrm{MeV}$ neutron capture line is not cospatial with the HXR continuum nor apparently even from a different part of the same loop. This seems to lead to the inescapable conclusion that ions are not accelerated in the same place as electrons though the two site must be linked in some way to allow 
for the observed degree of synchronism of the emissions (allowing for neutron capture delay). The only model offered so far is one by Miller (personal communication) based on his Alfven and fast mode turbulence acceleration model in which the electron/ion flux ratio depends on the size of the acceleration region.

\subsection{Particle Energy budget and the Neupert Effect}

As noted in Section 3, the number of energetic electrons involved in a flare is very large, comparable with all the electrons in a loop above the transition zone. This has given rise to the so-called electron number problem which is as persistent as it is mythical. There is no problem of adequate supply of electrons ! In any plasma, driven transfer of electrons from A to B automatically results in an electric field driving plasma charge drift from $\mathrm{B}$ to $\mathrm{A}$ so dense atmospheric drift currents replenish the electrons injected in any beam (Hoyng, Brown \& van Beek 1976; Knight \& Sturrock 1977; Colgate 1978). There may be problems as to return current stability, and of global current closure at the acceleration site but there is not a supply problem per se. On the other hand, even with the most conservative assumptions concerning $E_{c}$, thermal HXR contribution etc, there is little doubt that the power carried by fast electrons (and possibly also by ions though their distribution function is less well determined) is a significant fraction of flare impulsive phase power. Fast particles therefore play an important role in flare energy transport and plasma heating. There does not appear to be any mechanism other than electron bombardment able to deliver enough power to drive impulsive chromospheric flare emissions and to synchronise them and HXR emission from separate footpoints.

Another observation cited in support of flare plasma heating by energetic electrons is the Neupert Effect that, in many cases, the HXR light curve crosscorrelates quite well with the time derivative of the SXR light curve. Similar behaviour is reported for flare stars (Guedel et al. 2002) as discussed later in these proceedings. This data phenomenological effect is usually taken to mean that the fast electron power is deposited and accumulated in the (comparatively slow-cooling) SXR plasma. In fact it might only indicate a degree of synchronism between the electron acceleration process and a quite distinct plasma heating process, each associated with reconnection, with no direct heating by the beam. To check this, Veronig et al. (2003) have used RHESSI and GOES data to infer the actual total electron beam power $P_{e}(t)$ time variation (which depends on HXR flux, spectral index and $E_{c}$ ) and total power $P_{i n}(t)$ into the hot plasma required to drive the observed variations in its temperature and emission measure and to offset radiative and conductive losses. Physically, if electrons heat the plasma one would expect a much better cross correlation of $P_{e}(t)$ and $P_{i n}(t)$ than of the diagnostic HXR and SXR light curves. However, Veronig et al. find that, if $E_{c}$ is held fixed at any value, the cross-correlation is in fact worse between the physical (power) variables than between the phenomenological (photon flux) ones. This means either that fast electrons are not the dominant energy input into the hot plasma or that $E_{c}$ varies with time. Time variation of $E_{c}$ seems perfectly possible physically. This could arise in the acceleration process itself or be related to changes in the plasma temperature which, if high enough ('warm target') can change the 'effective' low energy cut-off (Emslie 2003). If one introduces the extra degree of freedom into the modelling parametrically in the 
form of a time varying low energy cut-off, i.e. $E_{c}=E_{c}(t)$, only a very modest variation in $E_{c}(t)$ (typically $25-30 \mathrm{keV}$ ) throughout the events is needed to allow exact matching $P_{e}(t)=P_{i n}(t)$ since $P_{e}$ is very sensitive to $E_{c}$ due to the steep electron spectra.

\section{Micro-events and Loop Mass Supply}

Increasing instrument sensitivity and resolution have revealed ever smaller and more transient events in the sun's atmosphere at all wavelengths. This leads us away from notions of quasi-steady quasi-spherical atmospheric heating and instead toward the idea of a hierarchical distribution of 'events' variously termed micro-/nano-events or -flares. The frequency distribution of these events over their energy release has been the subject of lively debate because the total power deposition in the solar atmosphere from such events depends critically on the slope of the event energy content distribution and on the minimum cutoff energy content. Certainly the behaviour of individual 'micro-events' in active region loops is quite flare-like, as most clearly seen in the movies of Krucker (2003) where one can see the sequence of low energy HXR footpoint brightenings, TRACE loop filling and plasma outflow, Type III burst and interplanetary plasma waves. Using SoHO EIT data, Brown et al. (2000) argued that the observed increase in temperature in such micro-events could not drive the observed increase in emission measure by conductive evaporation but required a nonthermal driver. They also argued that, if this driver were nonthermal electrons accelerated in coronal micro-flares, then one might expect to see quasicontinuous bursts of low energy $(\leq 10 \mathrm{keV})$ nonthermal HXR emission from the sun. RHESSI has discovered just such micro-burst HXR emission (Krucker et al. 2002, Lin 2003). However, the data have as yet to be subjected to quantitative physical modelling to see if the energy budget supports this idea and to see if the rate of mass evaporation is a significant contribution to mass supply to the general corona and wind.

\section{Some Remarks on Hot Star Magnetic Fields}

Contrary to previous expectations that hot stars would not have magnetic fields/activity, fields have now been detected directly in $\mathrm{O}$ and in Be stars at the level of hundreds of gauss (de Jong et al 2001; Neiner et al. 2003). It also seems theoretically that channelling of the wind by large scale dipole-like fields is the only way to explain the existence of dense Keplerian disks around Be stars (Cassinelli et al. 2002), a fact of possible relevance to protoplanetary disks. More speculatively, Waldron \& Cassinelli (2001) have proposed fast flows in solar-like 'coronal' magnetic loops as one explanation of the puzzling symmetry of X-ray line profiles from some hot stars. (Lines emitted in a spherical wind should be asymmetric due to the higher absorption of red wind photons from the receding hemisphere). MacGregor \& Cassinelli (2003) have discussed theoretically how such fields might be generated in hot stars. 


\section{Acknowledgements}

This work and my IAU participation were supported by funding from PPARC, The Royal Societies of London and Edinburgh, and by a NATO Collaborative Research Grant.

\section{References}

Ahmad, Q. R., Allen, R. C., Andersen,R. \& 176 coauthors 2002, Phys.Rev.Lett, 89,1301

Alexander, C. \& Brown, J.C. 2002, Solar Phys., 210, 407

Aschwanden, M.J., Brown J.C., \& Kontar, E. 2002, Solar Phys., 210, 383

Brown, J.C. 1971, Solar Phys., 18, 489

Brown, J.C., \& Melrose, D.B. 1976, Solar Phys., 52, 117

Brown, J.C., \& MacKinnon, A.L. 1985, ApJ, 292, L31

Brown, J.C., Kontar, E., \& Aschwanden, M.J. 2002, Solar Phys., 210, 373

Brown, J.C., Emslie, A.G., \& Kontar, E. 2003, ApJ, 595, L115

Cassinelli, J.P., Brown, J.C., Maheswaran,M., Millar, N., \& Telfer D. 2002, ApJ, 578,951

Colgate, S. A. 1978,ApJ, 221,1068

Conway A. J., Brown, J. C., Eves B., Kontar, E. P \& Schwartz, R. A. 2003, A\&A, 407, 725

Craig, I. J. D., \& Brown, J. C. 1986, Inverse Problems in Astronomy (Hilger)

Emslie, A. G. 2003,ApJ, 595, L119

Emslie, A. G., Barrett, R. K., \& Brown, J. C. 2001, ApJ, 557, 921

Fletcher, L. \& Hudson, H. S. 2002, Solar Phys., 210, 307

Gough, D. 2000, Science, 287, 2434

Guedel, M., Audard, M., Skinner, S. L., Horvath, M.I. 2002, ApJ, 580, L73

Haydock, E. L., Brown, J. C., Conway, A. J., \& Emslie, A. G. : 2001, Solar Phys. 203, 355

Holman, G. D., Sui, L., Schwartz, R. A., \& Emslie, A. G. 2003, ApJ, 595, L97

Hoyng, P., Brown, J. C., \& van Beek, H. F. 1976, Solar Phys., 48, 197

Hurford, G. J., Schwartz, R. A., Krucker, S., Lin, R. P., Smith, D. M., \& Vilmer, N. 2003, ApJ, 595,L77

de Jong, J. A., Henrichs, H. F., Kaper, L., Nichols, J. S., Bjorkman,J.E., \& 11 coauthors 2001, A\&A, 368, 601

Johns, C., \& Lin, R.P. 1992, Solar Phys, 137,121

Johns, C. and Lin, R.P. 2003, personal communication

Kane, S.R. 2003, SPD Abstract 34, 2206

Knight, J.W., \& Sturrock, P.A. 1977 ApJ, 218, 306

Kontar, E., Brown, J.C., Emslie, A.G., \& MacArthur, G.K. 2002, Solar Phys., 210,419 
Krucker, S., Christe, S., Lin, R. P., Hurford, G.J., Schwartz, R. A. 2002, Solar Phys., 210, 445

Krucker, S. http://sprg.ssl.berkeley.edu/ krucker/hessi/

Lin, R. P \& 57 co-authors 2002, Solar Phys., 210, 3

Lin, R. P. 2003, these proceedings

Litvinenko, Y. E. 2000, Solar Phys., 194, 327

MacGregor, K. B., \& Cassinelli, J. P., 2003, ApJ, 586, 480

Massone, A. M., Piana, M., Conway, A., \& Eves, B. 2003 , A\&A, 405, 325

Masuda, S. 1994, Nature, 371, 495

MacKinnon, A. L., Hayward, J., \& Brown, J. C. 1986, Solar Phys, 99, 231

Neiner, C., Hubert, A.-M., Frmat, Y., Floquet, M., Jankov, S., Preuss, O., Henrichs, H. F. \& Zorec, V. 2003, A\&A, 409, 275

Petkaki, P., \& MacKinnon, A.L. 1997, Solar Phys., 172, 279

Piana, M. 1994, A\&A, 288, 949

Piana, M., Brown, J. C., \& Thompson, A.M. 1995, Solar Phys., 156, 315

Piana, M., Massone, A. M., Kontar, E. P. Brown, J. C., \& Schwartz, R. A. 2003, ApJ, 595, L127

Scharmer, G. B., Gudiksen, B. V., Kiselman,D., Lofdahl, M. G., van der Voort, R.,\& Luc, H. M. 2002, Nature, 420, 151

Smith, D. M, Share, G. H., Murphy, R. J., Schwartz, R. A., Shih, A. Y., \& Lin, R. P. 2003, ApJ, 595, L81

Thomas, J. H., Weiss, N. O., Tobias, S. M., \& Brummell, N. H. 2002 Nature, 420, 390, 2002

Thompson, A. M., Brown J. C., Craig, I. J. D. \& Fulber, C. 1992, A\&A, 265, 278

Veronig, A. \& Brown, J. C. 2003, preprint

Veronig, A., Brown, J. C, Dennis, B. R., Sui, L., \& Tolbert, K. 2003, preprint

Waldron, W. L., \& Cassinelli, J. P. 2001, ApJ, 548, L45

Weiss, N. O. 2002 Astronomy \& Geophysics, 43, 9

Weiss, N. O. \& Tobias, S. M. 2002, Space Sci Rev., 94, 99 

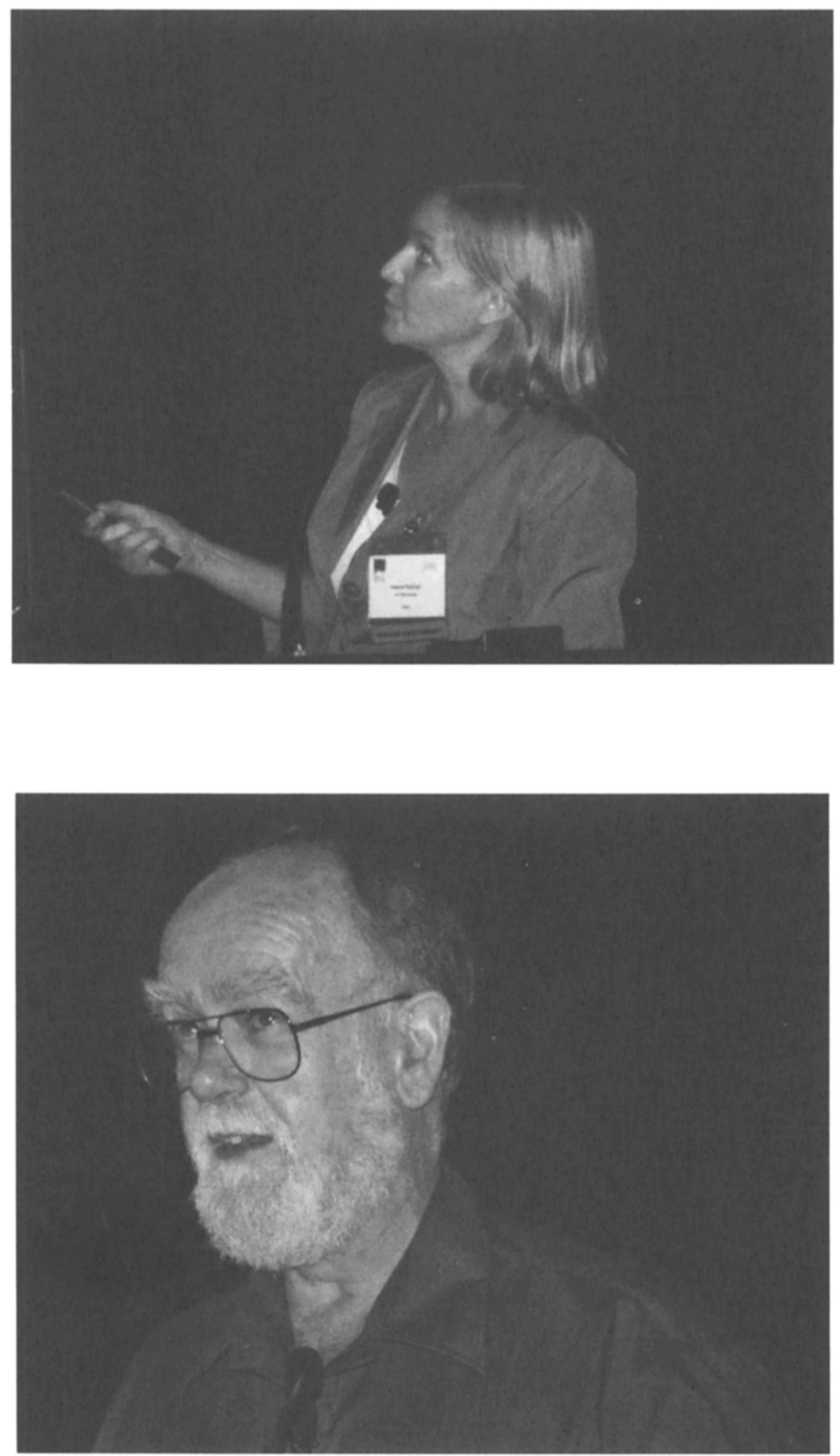